\title{
DECOMPOSITION OF TENSOR PRODUCTS OF IRREDUCIBLE UNITARY REPRESENTATIONS
}

\author{
DETLEV POGUNTKE
}

ABSTRACT. It is shown that the tensor product of an irreducible unitary representation of a (discrete) group $G$ and an $n$-dimensional $(n<\infty)$ unitary representation of $G$ decomposes into at most $n^{2}$ irreducible subrepresentations; the multiplicity of each irreducible constituent is not greater than $n$. As an application it is shown that the restriction of an irreducible unitary representation to a subgroup of finite index is a finite sum of irreducible subrepresentations.

At the meeting "Harmonische Analyse und Darstellungstheorie lokalkompakter Gruppen"' in Oberwolfach, R. Howe posed the following question: Let $G$ be a (discrete) group and let $\pi$ and $\rho$ be irreducible unitary representations of $G$ with $\operatorname{dim} \rho=: n<\infty$. Is $\rho \otimes \pi$ a sum of at most $n^{2}$ irreducible subrepresentations? He proved that the answer is yes if $\pi$ is finite dimensional, too. In this paper it is shown that the answer is yes in the general case. Indeed, we will show a little bit more, namely:

Theorem. Let $\pi$ and $\pi^{\prime}$ be irreducible unitary representations of the group $G$ in the Hilbert spaces $\mathcal{S}_{2}$ and $\mathcal{S}^{\prime}$, respectively, and let $\rho$ be a unitary representation of $G$ in a Hilbert space of dimension $n<\infty$. Then the dimension of the space $\operatorname{Hom}_{G}\left(\pi^{\prime}, \rho \otimes \pi\right)$ of intertwining operators is not greater than $n$ and is equal to $n$ iff $\rho \otimes \pi$ is unitarily equivalent to $n \pi^{\prime}$.

Corollary 1. Let $G, \rho, \pi, \sqrt{2}$ be as in the Theorem. Then the dimension of the algebra $\operatorname{Hom}_{G}(\rho \otimes \pi, \rho \otimes \pi)$ is not greater than $n^{2}$ and is equal to $n^{2}$ iff $\rho^{*} \otimes \rho \otimes \pi$ is unitarily equivalent to $n^{2} \pi$ ( $\rho^{*}$ denotes the contragradient representation). Especially, $\rho \otimes \pi$ is the direct sum of at most $n^{2}$ irreducible subrepresentations.

Corollary 2. Let $G, \pi, \sqrt{2}$ be as in the Theorem and let $N$ be a subgroup of $G$ of finite index. Then the restriction of $\pi$ to $N$ is a finite sum of irreducible subrepresentations. If $N$ is a normal subgroup the dimension of the algebra $\operatorname{Hom}_{N}(\pi, \pi)$ is not greater than the index $[G: N]$.

Corollary 2 was used in the proof of Proposition 2.1 in [1] for locally compact groups of type I.

The basic idea in the proof of the Theorem is the introduction of an inner

Received by the editors August 8, 1974 .

AMS (MOS) subject classifications (1970). Primary 22D10.

Key words and phrases. Irreducible unitary representations of groups, tensor products of representations, intertwining operators. 
product $\langle\langle\rangle$,$\rangle in \operatorname{Hom}_{G}(\rho \otimes \pi, \rho \otimes \pi)$ which is totally algebraic in nature. At the end of the paper, we will give an example of an $n$-dimensional (for all integers $n>1)$ irreducible representation $\rho$ such that $\rho \otimes \rho^{*}$ is a sum of $n^{2}$ one-dimensional subrepresentations.

Let $V$ be a finite dimensional Hilbert space and let $\sqrt{\zeta}$ be an arbitrary Hilbert space. The algebraic tensor product $V \otimes \sqrt[S]{ }$ becomes a Hilbert space if we define $\langle w \otimes k, v \otimes h\rangle=\langle w, v\rangle\langle k, h\rangle$. If $e_{1}, \ldots, e_{n}$ is an orthonormal basis of $V$, every element in $V \otimes \mathcal{F}_{2}$ can be represented uniquely in the form $\sum_{i=1}^{n} e_{i} \otimes h_{i}$ and, by definition of the inner product, we get

$$
\left\langle\sum_{i=1}^{n} e_{i} \otimes h_{i}, \sum_{i=1}^{n} e_{i} \otimes k_{i}\right\rangle=\sum_{i=1}^{n}\left\langle h_{i}, k_{i}\right\rangle .
$$

Now, we indicate how Corollary 1 (the answer to Howe's question) follows from the Theorem.

Let $\mathcal{S}^{\prime}$ be another Hilbert space and let $V^{*}$ denote the dual space of $V$. Then the spaces of bounded operators $\operatorname{Hom}\left(V \otimes \mathscr{F}_{2}, \mathscr{F}_{2}^{\prime}\right)$ and $\operatorname{Hom}\left(\mathscr{F}_{2}, V^{*} \otimes \mathscr{G}^{\prime}\right)$ are canonical isomorphic. The isomorphism $J: \operatorname{Hom}\left(V \otimes \mathcal{F}_{2}, \mathcal{F}_{2}^{\prime}\right) \rightarrow$ $\operatorname{Hom}\left(\mathcal{L}_{2}, V^{*} \otimes \mathcal{S}^{\prime}\right)$ is given by $(J T) h=\sum_{i=1}^{n} e_{i}^{*} \otimes T\left(e_{i} \otimes h\right)$ if $e_{1}^{*}, \ldots, e_{n}^{*}$ is the dual basis of $e_{1}, \ldots, e_{n} ; J T$ is independent of the basis $e_{1}, \ldots, e_{n^{*}} J$ is not an isometry for the operator norms, but we have

Lemma 1. $n^{-1 / 2}\|T\| \leq\|J T\| \leq n^{1 / 2}\|T\|$ for all $T \in \operatorname{Hom}\left(V \otimes \mathscr{F}, \mathcal{F}^{\prime}\right)$. More over, let $G$ be a group, and let $\rho, \pi$ and $\pi^{\prime}$ be unitary representations of $G$ in $V, \mathcal{F}_{2}$ and $\mathcal{S}_{2}^{\prime}$, respectively. Then $J$ transforms the space of intertwining operators $\operatorname{Hom}_{G}\left(\rho \otimes \pi, \pi^{\prime}\right)$ onto $\operatorname{Hom}_{G}\left(\pi, \pi^{*} \otimes \pi^{\prime}\right)$.

The simple proof of this lemma is omitted. For $\pi^{\prime}=\rho \otimes \pi$ we get $\operatorname{Hom}_{G}(\rho \otimes \pi, \rho \otimes \pi) \cong \operatorname{Hom}_{G}\left(\pi,\left(\rho^{*} \otimes \rho\right) \otimes \pi\right) \quad($ Theorem $\Rightarrow$ Corollary 1$)$.

Now, for a unitary representation $\rho$ in a finite dimensional Hilbert space $V$ and an irreducible unitary representation $\pi$ in a Hilbert $\delta$, we introduce an inner product $\left\langle\langle\right.$,$\rangle in \operatorname{Hom}_{G}(\rho \otimes \pi, \rho \otimes \pi)$ which is crucial in the proof of the Theorem (we will use that inner product for different $\rho$ 's and $\pi$ 's and will denote it by the same symbol $\langle\langle\rangle\rangle$,$) . We will compute \left\langle\left\langle T T^{*}, T T^{*}\right\rangle\right\rangle$ for $T \in$ Hom $_{G}\left(\pi^{\prime}, \rho \otimes \pi\right)$ in two different ways. The inner product is defined as follows:

If $S, T \in \operatorname{Hom}_{G}(\rho \otimes \pi, \rho \otimes \pi)$ then $J S, J T \in \operatorname{Hom}_{G}\left(\pi, \rho^{*} \otimes \rho \otimes \pi\right)$ and $(J S)^{*} J T \in \operatorname{Hom}_{G}(\pi, \pi)$. Since $\pi$ is irreducible, that operator is a scalar multiple of the identity, and we define

$$
\left\langle\langle T, S\rangle \mathrm{id}_{f_{2}}=(J S)^{*}(J T) .\right.
$$

Lemma 2. (i) 《, , is linear in the first and conjugate-linear in the second variable.

(ii) $\langle\langle S, S\rangle\rangle=\|J(S)\|^{2}$, especially, $\langle\langle S, S\rangle\rangle=0$ iff $S=0$. 


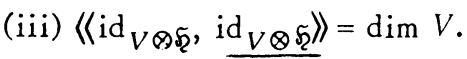

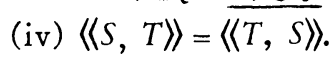

(v) Let $e_{1}, \ldots, e_{n}$ be an orthonormal basis of $V$, and let $S, T \in$ $\mathrm{Hom}_{G}(\rho \otimes \pi, \rho \otimes \pi)$ be given by

$$
S\left(e_{i} \otimes h\right)=\sum_{k=1}^{n} e_{k} \otimes S_{k i}(h) \quad \text { and } \quad T\left(e_{i} \otimes h\right)=\sum_{k=1}^{n} e_{k} \otimes T_{k i}(h)
$$

for $h \in \mathcal{S}_{2}$ and $1 \leq i \leq n$. Then the equation 《T, $S_{\| i \mathrm{~d}_{\mathscr{F}}}=\sum_{i, k=1}^{n} S_{i k}^{*} T_{i k}$ holds.

Proof. (i) and (ii) are clear, (iii) and (iv) follow from (v). Let $e_{1}^{*}, \ldots$, $e_{n}^{*}$ be the dual basis of $e_{1}, \ldots, e_{n}$. By definition, we have $(J S)(h)=$ $\sum_{i, k=1}^{n} e_{i}^{*} \otimes e_{k} \otimes S_{k i}(h)$. A simple computation shows that

and therefore,

$$
(J S)^{*}\left(\sum_{i, k=1}^{n} e_{i}^{*} \otimes e_{k} \otimes h_{k i}\right)=\sum_{i, k=1}^{n} S_{k i}^{*} h_{k i}
$$

$$
(J S)^{*}(J T)=\sum_{i, k=1}^{n} S_{i k}^{*} T_{i k}
$$

Definition. Let $\rho$ and $\pi$ be as before. Let $\pi^{\prime}$ be another irreducible unitary representation of $G$ in $\mathcal{L}^{\prime}$ and let $T \in \operatorname{Hom}_{G^{\prime}}\left(\pi^{\prime}, \rho \otimes \pi\right)$. The operator $T^{*} \in \operatorname{Hom}_{G}\left(\rho \otimes \pi, \pi^{\prime}\right)$ corresponds to an operator in $\operatorname{Hom}_{G}\left(\pi, \rho^{*} \otimes \pi^{\prime}\right)$ (by Lemma 1). This operator is denoted by $T^{a}$. Explicitly: if $e_{1}, \ldots, e_{n}$ is an orthonormal basis of $V$ with dual basis $e_{1}^{*}, \ldots, e_{n}^{*}$ and $T$ is represented as

$$
T h^{\prime}=\sum_{i=1}^{n} e_{i} \otimes T_{i} h^{\prime}, \text { then } T^{a} h=\sum_{i=1}^{n} e_{i}^{*} \otimes T_{i}^{*} h .
$$

Lemma 3. Let $0 \neq T \in \operatorname{Hom}_{G}\left(\pi^{\prime}, \rho \otimes \pi\right)$. Then $T^{*} T \in \operatorname{Hom}_{G}\left(\pi^{\prime}, \pi^{\prime}\right)$, $\left(T^{a}\right)^{*} T^{a} \in \operatorname{Hom}_{G}(\pi, \pi), T T^{*} \in \operatorname{Hom}_{G}(\rho \otimes \pi, \rho \otimes \pi)$ and $T^{a}\left(T^{a}\right)^{*} \epsilon$ . $\operatorname{Hom}_{G}\left(\rho^{*} \otimes \pi^{\prime}, \rho^{*} \otimes \pi^{\prime}\right)$. Let the positive real numbers $\alpha$ and $\beta$ be defined by $T^{*} T=\alpha \mathrm{id}_{f^{\prime}}$, and $\left(T^{a}\right)^{*} T^{a}=\beta \mathrm{id}_{f_{2}}$, respectively $\left(\pi^{\prime}\right.$ and $\pi$ are irreducible). Then one has

$$
\left\langle\left\langle T T^{*}, T T^{*}\right\rangle\right\rangle=\alpha \beta=\left\langle\left\langle T^{a}\left(T^{a}\right)^{*}, T^{a}\left(T^{a}\right)^{*}\right\rangle\right\rangle .
$$

Moreover, let $S: \mathcal{S}_{2}^{\prime} \rightarrow V \otimes \mathcal{F}_{2}$ be another intertwining operator such that the ranges of $S$ and $T$ are orthogonal. Then one has $\left\langle\left\langle S S^{*}, T T^{*}\right\rangle\right\rangle=0=\left\langle\left\langle S^{a}\left(S^{a}\right)^{*}\right.\right.$, $\left.\left.T^{a}\left(T^{a}\right)^{*}\right)\right\rangle$.

Proof. The first statements are clear. Let $e_{1}, \ldots, e_{n}$ be an orthonormal basis of $V$ and let $T$ be given by $T h^{\prime}=\sum_{i=1}^{n} e_{i} \otimes T_{i} h^{\prime}$. Then

$$
T^{*} T=\sum_{i=1}^{n} T_{i}^{*} T_{i}=\alpha_{\mathrm{id}} \mathscr{F}^{\prime}, \quad\left(T^{a}\right)^{*} T^{a}=\sum_{i=1}^{n} T_{i} T_{i}^{*}=\beta \mathrm{id}_{\xi_{2}}
$$

and 


$$
T T^{*}\left(e_{i} \otimes h\right)=\sum_{k=1}^{n} e_{k} \otimes T_{k} T_{i}^{*} h
$$

By Lemma 2(v), we get

$$
\left\langle\left\langle T T^{*}, T T^{*}\right\rangle\right\rangle_{\mathrm{id}_{\mathfrak{F}}}=\sum_{i, k=1}^{n}\left(T_{k} T_{i}^{*}\right)^{*} T_{k} T_{i}^{*}=\sum_{i, k=1}^{n} T_{i} T_{k}^{*} T_{k} T_{i}^{*}=\alpha \beta \mathrm{id}_{\gamma_{2}} .
$$

Since $\left(T^{a}\right)^{a}=T$ the same argument shows that $\alpha \beta=\left\langle\left\langle T^{a}\left(T^{a}\right)^{*}, T^{a}\left(T^{a}\right)^{*}\right\rangle\right\rangle$. Let $S$ be as in the lemma. From $T\left(\mathscr{S}^{\prime}\right) \perp S\left(\mathscr{\zeta}^{\prime}\right)$ we get $T^{*} S=0$ and, therefore,

$$
\sum_{i=1}^{n} T_{i}^{*} s_{i}=0
$$

Since $\left(T^{a}\right)^{*} S^{a} \in \operatorname{Hom}_{G}(\pi, \pi)$ there exists $\gamma \in \mathbf{C}$ such that

$$
\gamma \mathrm{id}_{\mathfrak{F}^{2}}=\left(T^{a}\right)^{*} S^{a}=\sum_{i=1}^{n} T_{i} S_{i}^{*}
$$

By Lemma 2(v):

$$
\left\langle\left\langle S S^{*}, T T^{*}\right\rangle\right\rangle \mathrm{id}_{\mathfrak{F}_{2}}=\sum_{i, k=1}^{n}\left(T_{k} T_{i}^{*}\right)^{*} S_{k} S_{i}^{*}=\sum_{i, k=1}^{n} T_{i} T_{k}^{*} S_{k} S_{i}^{*}=0 \quad \text { (by (1)). }
$$

Now, $T^{a}\left(T^{a}\right)^{*}$ (and, analogously, $\left.S^{a}\left(S^{a}\right)^{*}\right)$ is given by $T^{a}\left(T^{a}\right)^{*}\left(e_{i}^{*} \otimes h^{\prime}\right)=$ $\sum_{k=1}^{n} e_{k}^{*} \otimes T_{k}^{*} T_{i} h^{\prime}$. Lemma 2(v) implies:

$$
\left\langle\left\langle S^{a}\left(S^{a}\right)^{*}, T^{a}\left(T^{a}\right)^{*}\right\rangle\right\rangle_{\mathrm{id}}{\tilde{\xi^{\prime}}}=\sum_{i, k=1}^{n}\left(T_{k}^{*} T_{i}\right)^{*} S_{k}^{*} S_{i}=\sum_{i, k=1}^{n} T_{i}^{*} T_{k} S_{k}^{*} S_{i}=0
$$

(by (2) and (1)).

\section{Now we are able to give the}

Proof of the Theorem. Let $T_{1}, \ldots, T_{r}$ be $r$ linearly independent elements in $\operatorname{Hom}_{G}\left(\pi^{\prime}, \rho \otimes \pi\right)$. Without loss of generality we may assume that $T_{i}\left(\mathcal{S}^{\prime}\right) \perp T_{j}\left(\mathcal{S}^{\prime}\right)$ for $i \neq j$ (by orthogonalization with respect to the inner product $\left.(S, T) \mathrm{id}_{\mathscr{F}^{\prime}}=T^{*} S\right)$. We have to show that $r \leq n$. Let $\alpha_{i}, \beta_{i}, P_{i}$ and $Q_{i}$ $(1 \leq i \leq r)$ be defined by $T_{i}^{*} T_{i}=a_{i} \mathrm{id}_{\mathscr{F}^{\prime}},\left(T_{i}^{a}\right)^{*} T_{i}^{a}=\beta_{i} \mathrm{id}_{f_{2}}, P_{i}=\alpha_{i}^{-1} T_{i} T_{i}^{*}$ and $Q_{i}=\beta_{i}^{-1} T_{i}^{a}\left(T_{i}^{a}\right)^{*}$. Then the $P_{i}$ 's and $Q_{i}$ 's are projections in $\operatorname{Hom}_{G}(\rho \otimes \pi, \rho \otimes \pi)$ and $\operatorname{Hom}_{G}\left(\rho^{*} \otimes \pi^{\prime}, \rho^{*} \otimes \pi^{\prime}\right)$, respectively. By Lemma 3 , one has

and

$$
\left\langle\left\langle P_{i}, P_{i}\right\rangle\right\rangle=\beta_{i} a_{i}^{-1},\left\langle\left\langle Q_{i}, Q_{i}\right\rangle\right\rangle=\alpha_{i} \beta_{i}^{-1}
$$

$$
\left\langle\left\langle Q_{i}, Q_{j}\right\rangle\right\rangle=0=\left\langle\left\langle P_{i}, P_{j}\right\rangle\right\rangle \text { for } i \neq j .
$$

Let $P$ and $Q$ be defined by the equations $\mathrm{id}_{V \otimes \mathcal{F}_{2}}=P+\Sigma_{i=1}^{r} P_{i}$ and $\mathrm{id}_{V^{*} \otimes \mathscr{F}^{\prime}}=$ $Q+\Sigma_{i=1}^{r} Q_{i}$. From Lemma 2 , one can easily conclude that

$$
\left\langle\left\langle\mathrm{id}_{V \otimes \mathcal{F}_{2}}, P_{i}\right\rangle\right\rangle=\left\langle\left\langle P_{i}, P_{i}\right\rangle\right\rangle=\left\langle\left\langle P_{i}, \mathrm{id}_{V \otimes \mathcal{S}_{2}}\right\rangle\right\rangle
$$

and 


$$
\left\langle\left\langle\mathrm{id}_{V^{*} \otimes \mathfrak{F}^{\prime}}, Q_{i}\right\rangle\right\rangle=\left\langle\left\langle Q_{i}, Q_{i}\right\rangle\right\rangle=\left\langle\left\langle Q_{i}, \mathrm{id}_{V^{*} \otimes \mathcal{F}^{\prime}}\right\rangle\right\rangle
$$

(because the $P_{i}$ 's and $Q_{i}$ 's are projections). This shows that $\left\langle\left\langle P_{j}, P\right\rangle\right\rangle=0=$ $\left\langle\left\langle Q_{j}, Q\right\rangle\right\rangle$ for $1 \leq j \leq r$. Lemma 2 and the above relations imply

$$
\langle\langle P, P\rangle\rangle+\sum_{i=1}^{r} \beta_{i} \alpha_{i}^{-1}=n=\langle\langle Q, Q\rangle\rangle+\sum_{i=1}^{r} \alpha_{i} \beta_{i}^{-1} .
$$

Since $\langle\langle\rangle$,$\rangle is positive definite one has 2 n \geq \sum_{i=1}^{r}\left(\beta_{i} a_{i}^{-1}+\alpha_{i} \beta_{i}^{-1}\right)$. From $x+x^{-1} \geq 2$ for positive real numbers $x$ we get $r \leq n$ as desired.

If the dimension of $\operatorname{Hom}_{G}\left(\pi^{\prime}, \rho \otimes \pi\right)$ is equal to $n$, choose $n$ linearly independent elements $T_{1}, \ldots, T_{n}$ in that space with $T_{i}\left(\mathscr{S}_{2}^{\prime}\right) \perp T_{j}\left(\mathscr{F}^{\prime}\right)$ for $i \neq j$ and form $P_{i}$ and $P$ as above. It is easy to see that $\langle\langle P, P\rangle\rangle=0$ and, therefore, $P=0$ or $\mathrm{id}_{V \otimes \mathcal{F}_{2}}=\sum_{i=1}^{n} P_{i}=\sum_{i=1}^{n} \alpha_{i}^{-1} T_{i} T_{i}^{*}$. This shows that $V \otimes \mathcal{F}_{2}$ is the (orthogonal) sum of the $T_{i}\left(\mathcal{F}^{\prime}\right)$ 's but the restriction of the representation $\rho \otimes \pi$ to $T_{i}\left(\zeta^{\prime}\right)$ is unitarily equivalent to $\pi$ '. The "if-part" is trivial. The Theorem is proved, we have already pointed out how Corollary 1 follows from the Theorem.

Remark. The fact that $\rho \otimes \pi$ is the direct sum of at most $n^{2}$ irreducible subrepresentations can be proved quicker if one uses Lemma 1 and a similar trick as in the proof of the Theorem. More precisely, let $P_{1}, \ldots, P_{r}$ be orthogonal projections in $\operatorname{Hom}_{G}(\rho \otimes \pi, \rho \otimes \pi)$. As in the proof of the Theorem one gets $n \geq \Sigma_{i=1}^{r}\left\langle\left\langle P_{i}, P_{i}\right\rangle\right\rangle$. But $\left\langle\left\langle P_{i}, P_{i}\right\rangle\right\rangle=\left\|J\left(P_{i}\right)\right\|^{2}$ and $\left\|J\left(P_{i}^{i}\right)\right\| \geq$ $n^{-1 / 2}\left\|P_{i}\right\|=n^{-1 / 2}$ (Lemma 1 ) and, therefore, $n \geq r n^{-1}$ or $r \leq n^{2}$. Of course, the Theorem gives a more precise description.

Proof of Corollary 2. Since $\bigcap_{g \in G} g g^{-1}$ is a normal subgroup of finite index, it suffices to prove the second statement. The group $G$, resp. $H:=$ $G / N$, acts linearly on the space $\operatorname{Hom}_{N}(\pi, \pi)$ by $g \cdot f=\pi(g) f \pi(g)^{*}$. Let $\rho$ be any irreducible unitary representation of $H$ in the finite dimensional Hilbert space $E$; we consider $\rho$ as a representation of $G$, too. The space of intertwining operators $\operatorname{Hom}_{H}\left(\rho, \operatorname{Hom}_{N}(\pi, \pi)\right)=\operatorname{Hom}_{G}\left(\rho, \operatorname{Hom}_{N}(\pi, \pi)\right)$ is isomorphic to $\operatorname{Hom}_{G}(\rho \otimes \pi, \pi)$. By the Theorem, the dimension of that space is not greater than $\operatorname{dim} E$. Since $H$ is finite, every element in $\operatorname{Hom}_{N}(\pi, \pi)$ is contained in a finite dimensional $H$-invariant subspace of $\operatorname{Hom}_{N}(\pi, \pi)$. Therefore, the dimension of $\operatorname{Hom}_{N}(\pi, \pi)$ is not greater than $\Sigma_{\rho}(\operatorname{dim} \rho)^{2}, \rho$ being an equivalence class of irreducible unitary representations of $H$. But, by a well-known theorem in the representation theory of finite groups, the value of this sum is exactly the order of $H$.

Example. For all integers $n>1$ we will give an example of an $n$-dimensional irreducible representation $\rho$ and another irreducible representation $\pi$ such that $\rho \otimes \pi$ decomposes into exactly $n^{2}$ subrepresentations. To motivate our example let $\rho$ and $\pi$ be such representations, $\rho \otimes \pi=\bigoplus_{i=1}^{n^{2}} \pi_{i}$. Then the algebra $\operatorname{Hom}_{G}(\rho \otimes \pi, \rho \otimes \pi)$ is at least $n^{2}$ dimensional; from Corollary 1 we know that its dimension is exactly $n^{2}$. Therefore, the $\pi_{i}$ 's are uni- 
tarily nonequivalent. Moreover, again by Corollary $1, \rho^{*} \otimes \rho \otimes \pi$ is unitarily equivalent to $n^{2} \pi$; but on the other hand $\rho^{*} \otimes \rho \otimes \pi$ is equal to $\rho^{*} \otimes\left(\bigoplus_{i=1}^{n^{2}} \pi_{i}\right)$ $=\bigoplus_{i=1}^{n^{2}}\left(\rho^{*} \otimes \pi_{i}\right)$. Hence $\pi$ is unitarily equivalent to $\rho^{*} \otimes \pi_{i}$ for all $i$.

Let $G$ be the Heisenberg group over $\mathbf{Z} / n \mathbf{Z}$,

$$
G=\left\{\left(\begin{array}{lll}
1 & x & z \\
0 & 1 & y \\
0 & 0 & 1
\end{array}\right): x, y, z \in \mathbf{Z} / n \mathbf{Z}\right\} .
$$

The commutator subgroup $G^{\prime}$ is equal to the center

$$
Z G=\left\{\left(\begin{array}{lll}
1 & 0 & z \\
0 & 1 & 0 \\
0 & 0 & 1
\end{array}\right): z \in \mathbf{Z} / n \mathbf{Z}\right\}
$$

Let $\chi$ be a character of

$$
N:=\left\{\left(\begin{array}{lll}
1 & 0 & z \\
0 & 1 & y \\
0 & 0 & 1
\end{array}\right): y, z \in \mathbf{Z} / n \mathbf{Z}\right\}
$$

which is faithful on $Z G$ and let $\rho$ be the induced representation ind ${ }_{N}{ }_{i} X$ in the $n$-dimensional space $V . \rho$ is irreducible by the Frobenius reciprocity theorem: the restriction of $\rho$ to $N$ decomposes into $n$ different characters since $\chi$ is faithful on $Z G$. Choose $\pi=\rho^{*}$. For $g \in Z G$ we have

$$
\rho(g)=\chi(g) \mathrm{id}_{V}, \quad \pi(g)=\rho^{*}(g)=\overline{\chi(g)} \mathrm{id}_{V^{*}}
$$

and therefore,

$$
(\rho \otimes \pi)(g)=\chi(g) \mathrm{id}_{V} \otimes \overline{\chi(g)} \mathrm{id}_{V^{*}}=\mathrm{id}_{V \otimes V^{*}} .
$$

Hence the homomorphism $\rho \otimes \pi$ factors through $G / Z G$ which is abelian, and $\rho \otimes \pi$ decomposes into one-dimensional subrepresentations; $\rho \otimes \pi$ is the sum of all $n^{2}$ nonequivalent (see above) one-dimensional representations of $G$.

Acknowledgement. Originally, I proved the Theorem for $\pi=\pi^{\prime}$. The generalization was suggested by the referee. Moreover, the example is due to the referee; I gave an example only for $n=2$.

\section{REFERENCE}

1. C. C. Moore, Groups with finite dimensional irreducible representations, Trans. Amer. Math. Soc. 166 (1972), 401-410. MR 46 \#1960.

FAKULTÄT FÜR MATHEMATIK DER UNIVERSITÄT BIELEFELD, 48 BIELEFELD, KURT-SCHUMACHER-STRASSE 6, FEDERAL REPUBLIC OF GERMANY 